\title{
Integrative molecular bioinformatics study of human adrenocortical tumors: microRNA, tissue-specific target prediction, and pathway analysis
}

\author{
Zsófia Tömböl, Peter M Szabó, Viktor Molnár ${ }^{1}$, Zoltán Wiener ${ }^{1}$, \\ Gergely Tölgyesi ${ }^{1}$, János Horányi ${ }^{2}$, Peter Riesz ${ }^{3}$, Peter Reismann, \\ Attila Patócs ${ }^{4}$, István Likó ${ }^{5}$, Rolf-Christian Gaillard ${ }^{6}$, András Falus ${ }^{1,7}$, \\ Károly Rácz and Peter Igaz
}

\author{
Second Department of Medicine, Faculty of Medicine, Semmelweis University, Szentkirályi Street 46, \\ $\mathrm{H}-1088$ Budapest, Hungary \\ ${ }^{1}$ Department of Genetics, Cell- and Immunobiology, Faculty of Medicine, Semmelweis University, Nagyvárad Square 4, \\ $\mathrm{H}-1089$ Budapest, Hungary \\ ${ }^{2}$ First Department of Surgery and ${ }^{3}$ Department of Urology, Faculty of Medicine, Semmelweis University, Üllói Street 78./b, \\ $\mathrm{H}-1082$ Budapest, Hungary \\ ${ }^{4}$ Molecular Medicine Research Group, Hungarian Academy of Sciences and Semmelweis University, Szentkirályi Street 46, \\ $\mathrm{H}-1088$ Budapest, Hungary \\ ${ }^{5}$ Gedeon Richter Ltd, Gyömrői Street 19-21, H-1103 Budapest, Hungary \\ ${ }^{6}$ Division of Endocrinology, Diabetology and Metabolism, University Hospital Lausanne, Rue du Bugnon 46, CH-1011 Lausanne, \\ Switzerland \\ ${ }^{7}$ Research Group of Inflammation Biology and Immunogenomics, Hungarian Academy of Sciences and Semmelweis University, \\ Nagyvárad Square 4, H-1089 Budapest, Hungary \\ (Correspondence should be addressed to P Igaz; Email: igapet@bel2.sote.hu)
}

\begin{abstract}
MicroRNAs (miRs) are involved in the pathogenesis of several neoplasms; however, there are no data on their expression patterns and possible roles in adrenocortical tumors. Our objective was to study adrenocortical tumors by an integrative bioinformatics analysis involving miR and transcriptomics profiling, pathway analysis, and a novel, tissue-specific miR target prediction approach. Thirty-six tissue samples including normal adrenocortical tissues, benign adenomas, and adrenocortical carcinomas (ACC) were studied by simultaneous miR and mRNA profiling. A novel data-processing software was used to identify all predicted miR-mRNA interactions retrieved from PicTar, TargetScan, and miRBase. Tissue-specific target prediction was achieved by filtering out mRNAs with undetectable expression and searching for mRNA targets with inverse expression alterations as their regulatory miRs. Target sets and significant microarray data were subjected to Ingenuity Pathway Analysis. Six miRs with significantly different expression were found. miR-184 and miR-503 showed significantly higher, whereas miR-511 and miR-214 showed significantly lower expression in ACCs than in other groups. Expression of miR-210 was significantly lower in cortisol-secreting adenomas than in ACCs. By calculating the difference between $\mathrm{dCT}_{\text {miR-511 }}$ and $\mathrm{dCT}_{\text {miR-503 }}$ (delta cycle threshold), ACCs could be distinguished from benign adenomas with high sensitivity and specificity. Pathway analysis revealed the possible involvement of G2/M checkpoint damage in ACC pathogenesis. To our knowledge, this is the first report describing miR expression patterns and pathway analysis in sporadic adrenocortical tumors. miR biomarkers may be helpful for the diagnosis of adrenocortical malignancy. This tissue-specific target prediction approach may be used in other tumors too.
\end{abstract}

Endocrine-Related Cancer (2009) 16 895-906 


\section{Introduction}

Sporadic adrenocortical tumors are common, and their prevalence reaches up to 7-9\% in pathological series (Koch et al. 2002). Most of these are incidentally discovered, hormonally inactive benign tumors, whereas rare hormone-secreting adenomas (cortisolor aldosterone-secreting) and malignancies of the gland are associated with significant morbidity and mortality. The prognosis of adrenocortical cancer (ACC) is poor with an overall 5 year survival below $30 \%$ (Libè et al. 2007).

The molecular pathogenesis of sporadic adrenocortical tumors is not well elucidated. Bioinformatic approaches including transcriptomics and comparative genome hybridization analyses have revealed several gene expression and chromosome alterations that appear to be relevant in adrenocortical tumor pathogenesis (Igaz et al. 2006). To our knowledge, no studies investigating potential differences between microRNA (miR) expression patterns of various sporadic adrenocortical tumors have been published to date.

miRs are small non-protein-coding RNAs involved in the post-transcriptional negative regulation of gene expression. Mature single-stranded miRs of $\sim 22-24$ nucleotides (Ambros et al. 2003) specifically bind to complementary sequences in $3^{\prime}$-untranslated regions of mRNA molecules (Chen 2005). miRs may induce translational repression or target mRNA degradation (Lim et al. 2005). Recent investigations on simultaneous miR-induced expression changes in transcriptome and proteome have revealed mRNA degradation as the principal way of miR action in mammalian organisms (Baek et al. 2008, Selbach et al. 2008). It has therefore been proposed that biologically relevant miR targets should be identified by the simultaneous analysis of miR and mRNA expression patterns without the necessity of investigating protein expression with high-throughput methods (Grosshans \& Filipowicz 2008).

miR molecules are expressed in a tissue-specific fashion and may target various mRNAs in different organs. miRs have been implicated in the regulation of numerous cellular processes such as cell development, proliferation, differentiation, apoptosis (Wijnhoven et al. 2007, Bueno et al. 2008), and tumor development (Wijnhoven et al. 2007). Characteristic miR signatures were described in several non-endocrine (Barbarotto et al. 2008) and some endocrine tumors, e.g. follicular and papillary thyroid tumors (Weber et al. 2006), ovarian cancer (Yang et al. 2008), pituitary (Bottoni et al. 2007), and endocrine pancreatic tumors (Roldo et al. 2006).
The identification of potential miR targets is of pivotal importance for deciphering the molecular mechanisms of miR actions. Target selection can be performed by publicly available web-based databases using different computational target prediction algorithms. The most extensively applied databases include miRBase (Griffiths-Jones et al. 2008), TargetScan (Grimson et al. 2007), and PicTar (Krek et al. 2005). Since there is no unambiguous preference for any of these target prediction databases at present, target selection was performed arbitrarily by using a preferred algorithm in most studies. In silico target prediction algorithms take several biophysical, biochemical, and evolutionary parameters into consideration (Krek et al. 2005, Grimson et al. 2007, Griffiths-Jones et al. 2008); however, the predicted number of potential miR-mRNA interactions is generally huge and no data can be inferred on their biological relevance. Owing to the lack of objective target selection methods and the difficulties of experimental target validation, very few target mRNAs could be validated as posttranscriptionally regulated by specific miRs to date.

In this study, adrenocortical tumors have been examined by an integrative molecular and bioinformatic approaches including both miR and mRNA profiling. Since the selection of biologically relevant miR targets is unsolved at present, we introduced a novel, tissue-specific miR target prediction approach by using the results of a parallel transcriptomics analysis. In order to achieve tissue specificity, undetectable mRNAs were subtracted from the pool of potential miR targets and we searched for mRNAs with inverse expression alterations as their regulatory miRs. By this approach, the pool of potential miR targets can be reduced and biologically relevant targets may be selected. For this purpose, a novel dataprocessing software was developed (the experimental workflow is presented in Fig. 1). Combining these data with pathway analysis revealed important pathomechanisms involved in adrenocortical tumorigenesis.

\section{Materials and methods}

\section{Tissue samples}

Thirty-six tissue samples were studied: ten normal adrenocortical tissues (two males and eight females; mean age at surgery: 53.4 years), ten hormonally inactive adenomas (IA) (all females; mean age: 50.4 years), nine cortisol-producing adenomas (CPA) (two males and seven females; mean age: 40.16 years), and seven ACCs (two males and five females; mean age: 54.90 years) were studied. The study was 


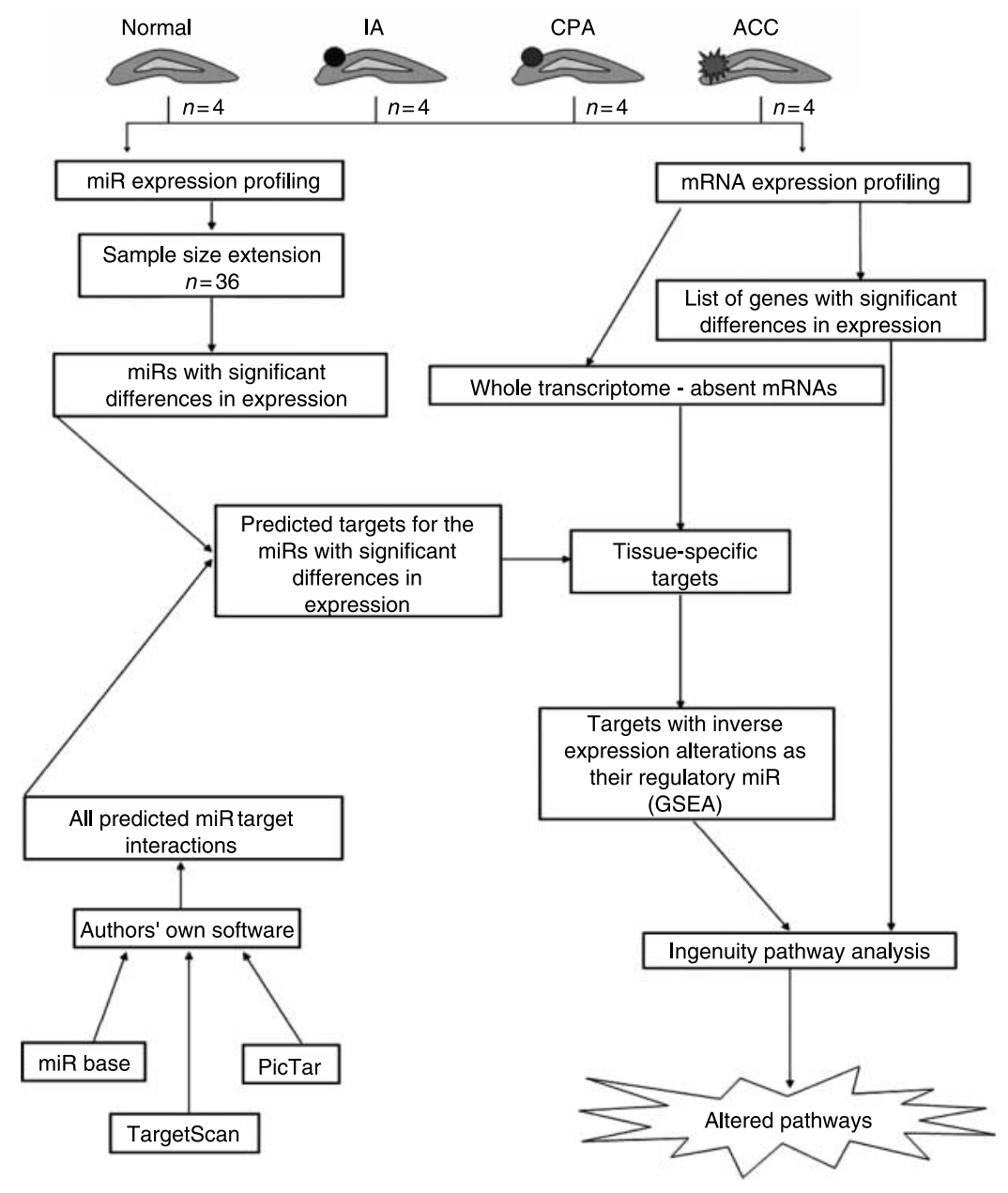

Figure 1 Illustration of the experimental workflow. IA, inactive adenoma; CPA, cortisol-producing adenoma; ACC, adrenocortical carcinoma.

approved by the Ethical Committee of the Hungarian Health Council, and informed consent was obtained from all patients involved. All ACCs were cortisol secreting. Normal adrenocortical tissues were obtained from patients undergoing total nephrectomy for kidney tumors. Tissue samples were immediately snap frozen in liquid nitrogen after removal and stored at $-80{ }^{\circ} \mathrm{C}$ until RNA isolation.

\section{miR expression profiling and quantitative reverse transcription-PCR}

Simultaneous miR and mRNA profiling was performed in 16 samples (four from each group). miRs showing significant differences in expression were examined in all tissue samples by quantitative reverse transcriptionPCR (qRT-PCR).

Total RNA was isolated by TriReagent (Molecular Research Center Inc., Cincinatti, OH, USA) for
miR expression profiling and qRT-PCR experiments. RNA integrity was verified by Agilent 2100 Bioanalyzer System (Agilent Tech. Inc., Santa Clara, CA, USA).

Total RNA of $100 \mathrm{ng}$ per sample was reverse transcribed by TaqMan MicroRNA Reverse Transcription Kit and Multiplex RT for TaqMan MicroRNA Assays (all materials from Applied Biosystems, Foster City, CA, USA) according to the manufacturer's instruction.

miR expression profiling of $368 \mathrm{miRs}$ was performed on Taqman Low Density Array (TLDA) Human MicroRNA Panel v1.0 (Applied Biosystems) according to the manufacturer's protocol using 7900HT Fast Real-Time PCR System (Applied Biosystems).

Normalized signal levels for each $\mathrm{miR}$ were calculated using comparative cycle threshold method (ddCT method; Livak \& Schmittgen 2001) relative to $R N U 48$ following the manufacturer's instruction 
(SDS programme, Applied Biosystems). Further data analysis was performed by BRB-ArrayTools Version 3.6.0 Stable Release (developed by Dr Richard Simon and Amy Peng Lam).

Differentially expressed miRs were identified by one-way ANOVA $(P<0.05)$ using SPSS 15 for Windows statistical software (SPSS Inc., Chicago, IL, USA), followed by the conservative Scheffé post hoc test. In order to study only miRs of biological relevance further, by using an arbitrary filter criterion, miRs unexpressed in at least $50 \%$ of the samples studied by TLDA Human MicroRNA Panel were filtered out.

Fourteen miRs with significant differences $(P<0.05)$ in expression revealed by TLDA Human MicroRNA Panel were selected for further validation and sample size extension by qRT-PCR. miRs with the most significant $(P<0.01)$ differences in expression $(10 \mathrm{miRs})$ and another four significant $(P<0.05)$ miRs passing an arbitrary fold change filtering criterion (higher than threefold significant expression change) were selected for qRT-PCR analysis. Taqman MicroRNA Assays containing unique miR-specific RT primers were used as follows: $m i R-181 b$ (4373116), $m i R-181 d$ (4373180), miR-184 (4373113), miR-196b (4395326), miR-210 (4373089), miR-214 (4395417), miR-215 (4373084), miR-222 (4395387), miR-375 (4373027), miR-424 (4373201), miR-503 (4373228), miR-506 (4373231), miR-511 (4373236), miR-615 (4380991), and RNU48 (4373383) as housekeeping genes (all materials from Applied Biosystems). qRT-PCR was performed using TaqMan Fast Universal PCR Master Mix on a 7500 Fast Real-Time PCR System according to the manufacturer's protocol. Data analysis was performed as above detailed using the SDS programme.

For qRT-PCR measurements, the same statistical analysis was performed as for the TLDA Human MiR Panel. In order to identify miR markers applicable for diagnosis, receiver-operating characteristics (ROC) analysis was performed by SPSS software.

\section{mRNA expression profiling}

Whole-genome mRNA expression analysis was performed on the same samples as used for miR profiling using $4 \times 44 \mathrm{~K}$ Whole Human Genome Microarrays (Agilent Tech. Inc). Microarray experiments were done in a two-colour experimental design by comparing individual samples to a uniform reference pool of all samples examined.

For the mRNA microarray analysis, total RNA was isolated from homogenized tissue samples using RNeasy Lipid Tissue Mini Kit (Qiagen GmbH).
RNA integrity was verified by Agilent 2100 Bioanalyzer System (Agilent Tech. Inc).

Total RNA of $1 \mu \mathrm{g}$ mixed with RNA Spike-In Kit (Agilent Tech. Inc.) was reverse transcribed, and cyanine 5 or cyanine 3-CTP-labeled (Perkin-Elmer, Waltham, MA, USA). cRNA was synthesized using the Low RNA Input Linear Amplification Kit (Agilent Tech. Inc.) according to the manufacturer's Dual Color Expression Microarray Protocol version 5.5. Successful labeling, cRNA yield, and purity were controlled on a Nanodrop ND-1000 Spectrophotometer (Thermo Scientific, Wilmington, DE, USA).

Cyanine 5-CTP-labeled cRNA of 825 ng per sample was mixed with an equal amount of cyanine 3-CTPlabeled uniform reference cRNA, and hybridized to $4 \times 44 \mathrm{~K}$ Whole Human Genome Microarrays (Agilent Tech. Inc.) according to the manufacturer's protocol.

Array scanning, feature extraction, and data normalization were performed with Agilent DNA Microarray Scanner and Feature Extraction Software 8.5 (Agilent Tech. Inc.) with default normalization scenario for Agilent $4 \times 44 \mathrm{~K}$ two-colour array platforms.

Statistical analysis of mRNA microarray data was performed by Genespring GX 9.0 Software (Agilent Tech. Inc.) with default scenario. Identification of gene sets differentially expressed in microarray analysis was carried out by one-way ANOVA $(P<0.05)$ followed by Benjamini-Hochberg multiple testing correction. Tukey's Honestly Significant Difference test (Tukey's HSD) was applied as standard multiple comparison test for microarray data analysis (Pavlidis 2003, Hulshizer \& Blalock 2007).

\section{Tissue-specific miR target prediction}

Identification of potential miR target molecules was performed using TargetScan 4.2 (http://www.targetscan. org), PicTar (http://pictar.org), and miRBase Targets Version 5 (http://microrna.sanger.ac.uk) databases.

The outputs of all three databases were merged and all potential targets of a miR predicted by these databases were identified by an own software application built with Microsoft Visual FoxPro 9.0 (Microsoft Inc.) developer system. This data-processing software is capable of converting the different target identifiers (HUGO Gene ID, Ensembl Transcript ID and RefSeq ID) applied by these prediction algorithms to each other. The annotation database was downloaded from the Ensembl homepage (www.ensembl.org). The software is able to identify 
all predicted target mRNAs for a miR or miR set, to provide score values retrieved from the different algorithms, and to mark mRNA targets overlapping in two or more databases.

As mRNAs not co-expressed with their regulatory miRs cannot be affected by miR regulation, these transcripts were filtered out from the list of potential targets by Microsoft Visual FoxPro 9.0. Arbitrary filtering criteria for subtracting absent mRNAs from microarray lists were the following: the percentage of 'present flag' and raw signal values higher than 50th percentile (of all raw signal values measured in the same sample) in less than three out of four samples in each experimental group. The mean of the 50th percentiles of raw signal values in the 16 samples studied considerably exceeded the background signal values.

We used Gene Set Enrichment Analysis (GSEA; Subramanian et al. 2005) to select potential mRNA targets with inverse expression alterations as their regulatory miRs. As a traditional gene expression analysis focuses on genes with significant differences in expression, biologically relevant, but fine alterations of gene expression data may remain unnoticed. GSEA is an alternative computational method developed for the analysis of these fine alterations. GSEA determines whether a priori or a user-defined set of genes shows statistically significant, concordant differences between two biological states (e.g. phenotypes). GSEA analyses gene expression data by rank statistics, and determines whether a particular set of genes is over- or underrepresented in the samples compared (Subramanian et al. 2005).

We used a novel GSEA approach to analyze potential, tissue-specific target sets of miRs with significant differences in expression alongside transcriptomic results, and to select potential mRNA targets with inverse expression alterations as their regulatory miRs. The enrichment analysis was performed by GSEA Software v2.0 (www.broad.mit.edu).

User-defined gene sets were created from tissuespecific filtered target lists of each miR with significant differences in expression in all pairwise comparisons of the groups studied. To reduce the amount of falsenegative miR target interactions, a target set of a given miR contained those predicted tissue-specific targets, which were found to be expressed in at least one of the two compared experimental groups. Five miR target sets in normal versus ACC, four in IA versus ACC, four in CPA versus ACC, and one in normal versus CPA comparisons were established.

By GSEA, microarray data were rank ordered in a stepwise fashion based on differences in expression between the two groups compared. From the extremes (top or bottom: the most up- and downregulated mRNAs respectively) of these rank-ordered entire lists of microarray data, potential miR targets showing inverse expression alterations as their regulatory miRs could be selected. If a $\mathrm{miR}$ is underexpressed, its potential targets are supposed to be upregulated. Therefore, targets located at the top of ranked lists were selected by setting a cut-off at the maximal positive deviation of running enrichment score from zero. In a similar fashion, potential targets of overexpressed miRs are assumed to be downregulated, and therefore targets located at the bottom of the ranked list should be selected. This selection was performed by setting the threshold of the running enrichment score at the maximal negative deviation from zero.

\section{Pathway analysis}

GSEA-filtered tissue-specific miR targets and significant mRNA microarray results in all pairwise comparisons were analyzed using Ingenuity Pathway Analysis (IPA) 7.1 Software (Ingenuity Systems, Redwood City, CA, USA; www.ingenuity.com).

\section{Results}

\section{miR expression analysis and biomarkers}

Twenty-two miRs showed significant $(P<0.05)$ differences in expression among the experimental groups studied by TLDA Human MiR Panel. miR-184 was detected only in ACC tissues. Both significant miRs and samples were then classified by hierarchical clustering based on similarities in expression profiles (Fig. 2).

Fourteen miRs were selected for further validation and sample size extension. By qRT-PCR, significant differences in expression were confirmed for six miRs $(m i R-184, \quad m i R-210, m i R-214, m i R-375$, $m i R-503$, and $m i R-511)$. The success rate for validating high-throughput TLDA measurements by qRT-PCR ( 6 out of $14 \mathrm{miRs}$ ) is similar to that previously reported by Resnick et al. (2009).

Significantly higher expression levels of $m i R-184$ and $m i R-503$ were detected in ACCs in comparison with their normal and benign counterparts, while $m i R-210$ expression was significantly higher in ACCs compared with CPAs. Expression of $m i R-511$ and $m i R-214$ was significantly lower in ACCs relative to other experimental groups. On the other hand, expression of $m i R-375$ was significantly higher in normal tissues compared with ACCs and CPAs (Fig. 3A and B). 


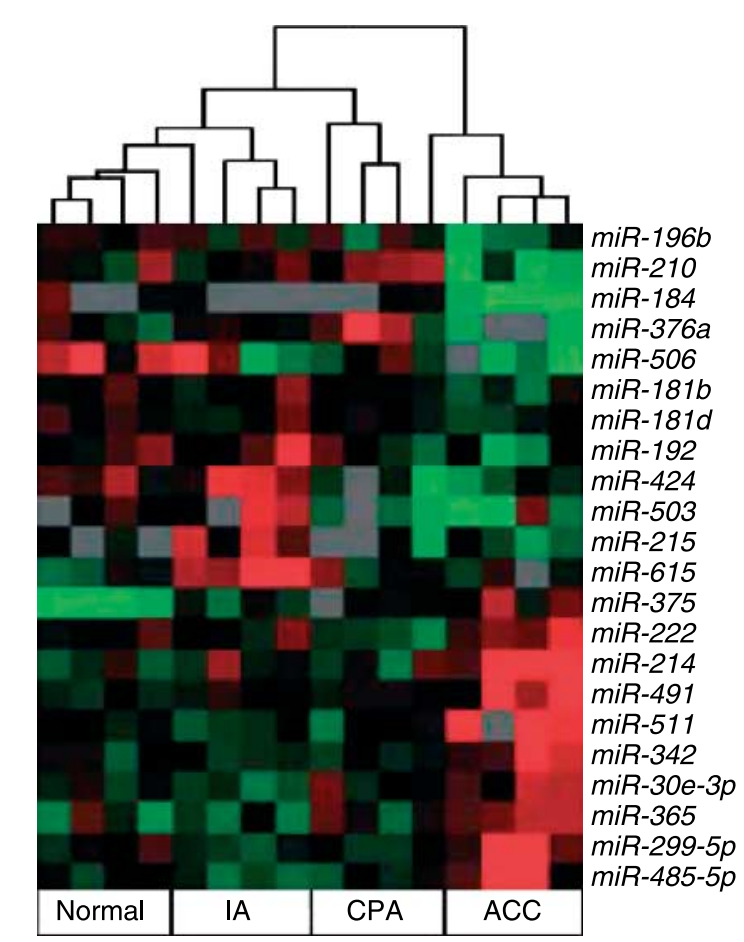

Figure 2 Hierarchical clustering of 16 different adrenocortical samples based on their miR expression patterns. Twenty-two miRs with significant differences in expression were subjected to hierarchical clustering by BRB-Array Tools Version 3.6.0 Stable Release. dCT values of these miRs relative to $R N U 48$ were clustered after mean centering the dCT data for each miR and for the experimental samples. Genes and samples were both clustered by centroid linkage hierarchical clustering method. Overexpression is indicated with red, whereas underexpression is coded with green.

To identify potential miR biomarkers of malignancy, miRs with the most significant delta CT (dCT) differences between ACCs and the other tissues ( $m i R-184, m i R-503$, and $m i R-511)$ and the combination of these miR pairs were tested by ROC analysis (Fig. 3C). Setting the cut-off value of $\mathrm{dCT}_{\text {miR-511- }}$ $\mathrm{dCT}_{\text {miR-503 }}$ to 1.4 (diagnosis is ACC if the difference is $\geq 1.4$ ), ACCs can be identified with $100 \%$ sensitivity and $97 \%$ specificity. By setting the cut-off value of $\mathrm{dCT}_{\text {miR-511 }}$ to $8.5,100 \%$ sensitivity and $93 \%$ specificity could be achieved, while at a cut-off value of -6.45 for $\mathrm{dCT}_{\mathrm{miR}-511}-\mathrm{dCT}_{\mathrm{miR}-184}$, ACCs can be distinguished from benign samples with $100 \%$ sensitivity and $80 \%$ specificity.

\section{Whole-genome mRNA expression profiling}

Altogether 614 genes showed significant differences in expression among the groups studied $(P<0.05)$, significant microarray data are presented in the electronic Supplementary Table 1, which can be viewed online at http://erc.endocrinology-journals. org/supplemental/. In the ACC versus normal comparison, 302 genes were upregulated, whereas 301 were found to be downregulated in cancer tissues. All results are accessible at Gene Expression Omnibus (www. ncbi.nlm.nih.gov/geo, accession number: GSE14922).

Several genes previously described as differentially expressed in already reported adrenocortical tumor microarrays have been revealed (Soon et al. 2008, Giordano et al. 2009). Topoisomerase II- $\alpha$ (TOP2A) expression was higher than ninefold in ACCs compared with other groups. Insulin-like growth factor 2 (IGF2) mRNA level was almost sevenfold higher in ACCs relative to IAs and threefold higher relative to CPAs and normal tissues. Overexpression of IGF2 is regarded as one of the most characteristic molecular alterations in ACC (Igaz et al. 2006, Soon et al. 2008, Giordano et al. 2009). Cyclin B2 (CCNB2) expression was at least 14-fold higher in ACCs than in any other tissue studied. Cell division cycle 2 and 25C (CDC2 and $C D C 25 C$ ) expression was also significantly higher in ACCs relative to the normal cortex and benign adenomas. On the other hand, cyclin-dependent kinase inhibitor 1C (CDKN1C) expression was significantly lower in ACCs relative to normal tissues.

\section{Tissue-specific miR target prediction}

Identification of miR target molecules was carried out using three web-based target prediction algorithms: TargetScan, miRBase, and PicTar. The outputs of these three algorithms were merged and analyzed by an own novel software application. The major advantage of our data-processing software is its potential for comparing the outputs of these algorithms, and identifying the overlapping targets predicted by two or more databases. Moreover, it provides an unfiltered list of all potential targets predicted by TargetScan, miRBase, and PicTar, which can be filtered further.

A total of 17868 mRNA molecules have been predicted to be targets of the 6 miRs with significant differences in expression. Since one mRNA molecule can be targeted by more than one miR, this means only 13147 different targets. Altogether, 7140 mRNAs were predicted by miRBase, 667 by PicTar, and 11336 mRNAs by TargetScan. For the 6 miRs studied, 1275 overlapping targets could be identified, which amounts to $7 \%$ of all predicted targets (Table 1).

In order to reduce the false-positive predictions and to identify biologically relevant miR targets in adrenocortical tissues, we developed a novel tissuespecific target prediction approach by applying the results of the three target prediction algorithms and the simultaneously performed mRNA microarray 
A

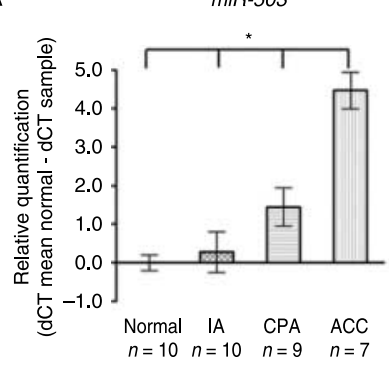

B

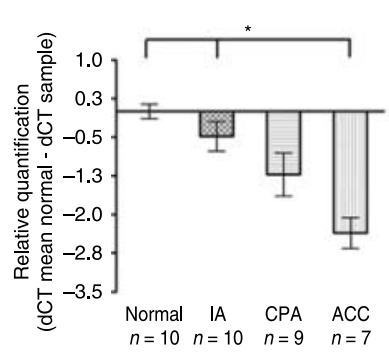

C

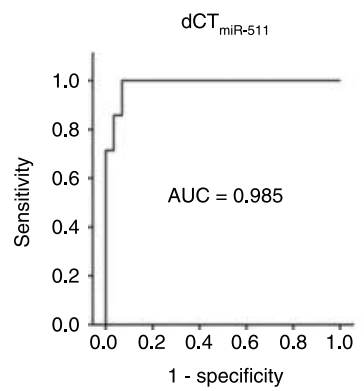

$\operatorname{miR}-184$

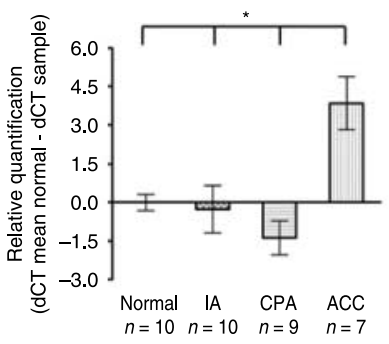

miR-511
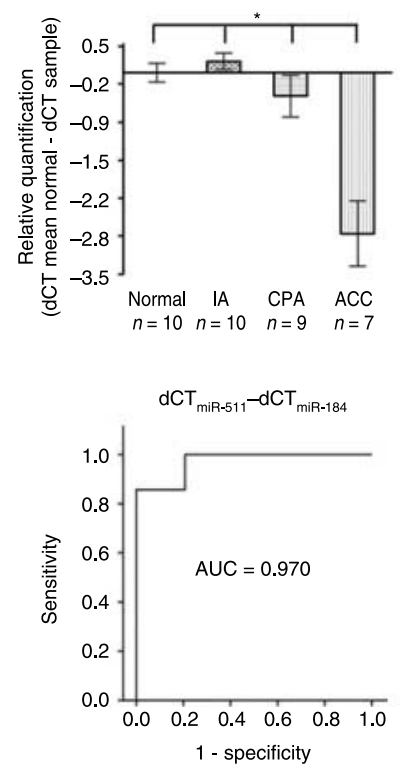

$\operatorname{miR}-210$
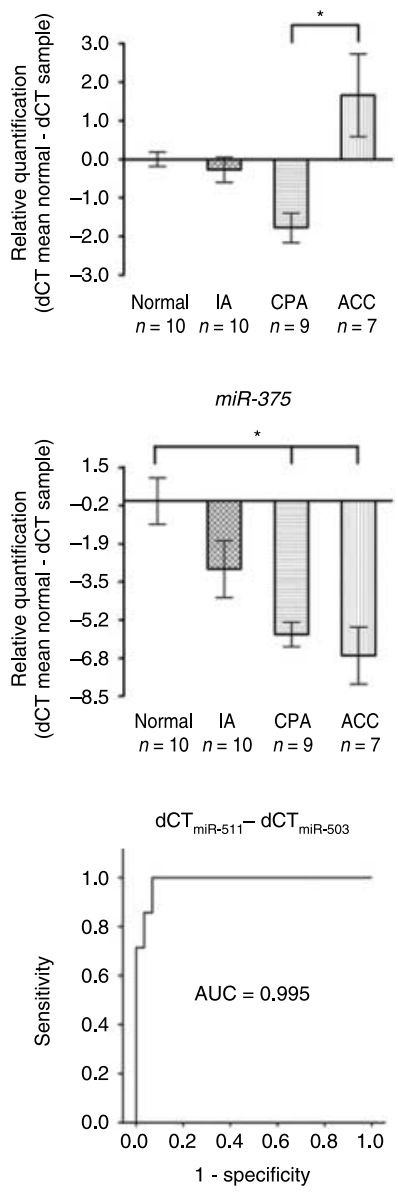

Figure 3 qRT-PCR results of six miRs with significant differences in expression represented by ddCT values relative to the mean of normal samples (mean \pm s.E.M). ${ }^{\star} P<0.05$. (A) Significantly overexpressed, (B) significantly underexpressed miRs in ACCs.

(C) ROC curves for the three most appropriate biomarkers for distinguishing ACCs from benign adrenocortical tumors.

measurements. As mRNAs not co-expressed with their regulatory miRs cannot be relevant targets of miR-mediated regulation, gene products absent from the tissues studied were excluded from further analysis. The number of predicted miR targets could be considerably reduced by using the filtered sets of mRNAs (Table 1).

Recent reports (Baek et al. 2008, Grosshans \& Filipowicz 2008, Selbach et al. 2008) have suggested that miR-evoked changes of protein level are well

Table 1 Numbers of potential targets for the six miRs with significant differences in expression predicted by TargetScan, PicTar, and miRBase and the number of tissue-specific targets expressed in human normal adrenal cortex, IA, CPA, and ACC samples

\section{All predicted tissue-specific targets}

\begin{tabular}{lcccccrrr} 
miR ID & TargetScan & PicTar & miRBase & $\begin{array}{c}\text { All predicted } \\
\text { targets }\end{array}$ & $\begin{array}{c}\text { Normal adrenal } \\
\text { cortex }\end{array}$ & IA & CPA & ACC \\
\hline miR-184 & 609 & 17 & 1164 & 1683 & 771 & 906 & 905 & 909 \\
miR-503 & 1547 & NP & 1225 & 2617 & 1359 & 1642 & 1635 & 1573 \\
miR-511 & 3045 & NP & 1064 & 3945 & 2295 & 2615 & 2658 & 2536 \\
miR-214 & 3874 & 528 & 1427 & 5252 & 2870 & 3463 & 3470 & 3367 \\
miR-375 & 1790 & 107 & 1158 & 2871 & 1537 & 1781 & 1804 & 1744 \\
miR-210 & 471 & 15 & 1102 & 1500 & 687 & 793 & 807 & 784 \\
\hline
\end{tabular}

IA, hormonally inactive adenoma; CPA, cortisol-producing adenoma; ACC, adrenocortical carcinoma; NP, non-predicted. 
reflected by mRNA expression alterations. mRNA degradation is thus considered as the principal way of miR action. To select the tissue-specific target mRNAs regulated by miRs mainly via target degradation, a novel GSEA approach was performed. By GSEA, we searched for target mRNAs showing inverse expression alterations compared with their potential regulatory miR molecules, as a further step to achieve tissue specificity. GSEA approach revealed 1306 targets for the significant miRs in normal versus ACC, 647 in IA versus ACC, 570 in CPA versus ACC, and 451 in normal versus CPA comparisons.

\section{Pathway analysis}

miR target sets revealed by GSEA in all pairwise comparisons and significant mRNA microarray data have both been subjected to IPA to identify potential pathogenetic pathways involved in adrenocortical tumorigenesis.
IPA revealed several possible pathways altered at the transcriptional level or potentially modified by miRs at the post-transcriptional level. Both IPA approaches (miR target mRNAs and gene transcripts with significant differences) in ACC to normal comparison revealed 'cell cycle: G2/M DNA damage checkpoint regulation' (Fig. 4, electronic Supplementary Tables 2 and 3, which can be viewed online at http://erc.endocrinology-journals.org/supplemental/) as the top canonical pathway.

\section{Discussion}

miRs expressed in malignant tumors have been classified as tumor suppressors and oncogenes based on their relative under- or overexpression (Chen 2005). The overexpressed miRs in ACC (miR-503, miR-210, and $m i R-184)$ may thus be regarded as oncogenes, whereas the underexpressed $(m i R-511, m i R-214$, and $m i R-375)$ may be tumor suppressors in ACC.

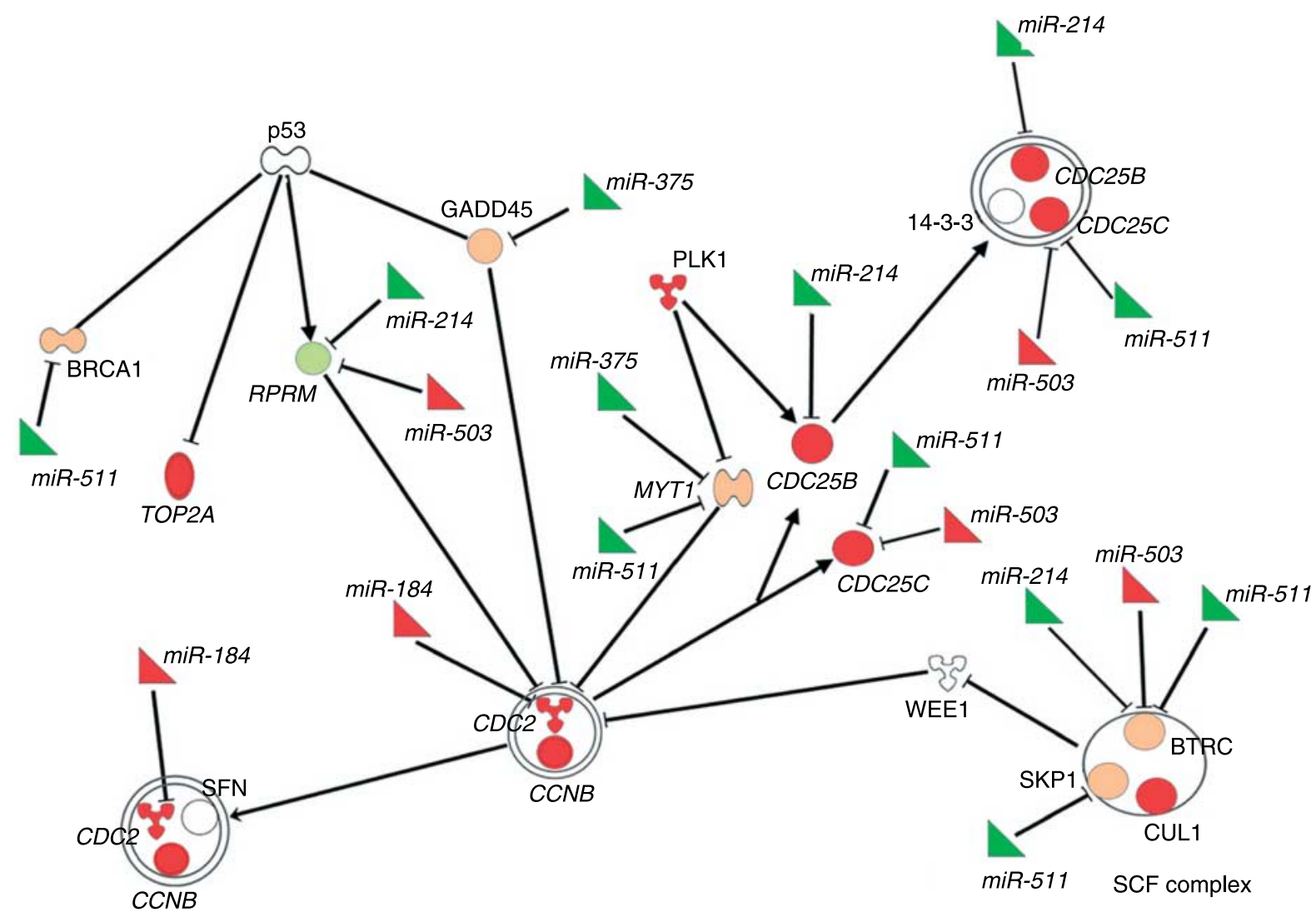

Figure 4 Cell cycle: G2/M DNA damage checkpoint regulation, as the top canonical pathway altered in ACCs. Lines between the proteins represent known interactions. Triangles represent miRs: red, significantly overexpressed; pink, non-significantly overexpressed; light green, non-significantly underexpressed; green, significantly underexpressed genes. 
Overexpression of $m i R-503$ has already been described in human retinoblastoma samples relative to normal retinal tissues; however, its exact role in tumorigenesis is unclear (Zhao et al. 2009). Overexpression of $m i R-210$ has been reported in breast cancer, and hypoxia may be a major factor in inducing $m i R-210$ expression (Camps et al. 2008). Hypoxia is also a feature of malignant adrenocortical neoplasms (Bernini et al. 2002). $m i R-184$ overexpression was noted in squamous cell carcinoma of the tongue (Wong et al. 2008).

$m i R-375$ was found to be significantly underexpressed in both benign and malignant hepatocellular neoplasms associated with $\beta$-catenin mutations relative to their wild-type counterparts. Direct interactions between activated $\beta$-catenin and $m i R$ - 375 are supposed (Ladeiro et al. 2008). Wnt- $\beta$-catenin signaling has been implicated in the pathogenesis of ACC (Soon et al. 2008); therefore, the underexpression of $m i R-375$ in our ACC samples may support the relevance of this pathway. In both groups of cortisol-secreting tumors (CPA and ACC), low expression of miR-375 was found.

In vitro inhibition of $m i R-214$ decreased, whereas inhibition of $m i R-210$ increased apoptosis of HeLa cells (Cheng et al. 2005). miR-184 has also been supposed to act via the regulation of apoptosis (Wong et al. 2008). The relative overexpression of $m i R-210$ and $m i R-184$ and underexpression of $m i R-214$ in our ACC samples may thus be associated with reduced apoptosis that is a common feature of malignancy. In human ovarian cancer, however, $m i R-214$ was regarded as an oncogenic miR based on increased expression via targeting phosphatase and tensin homolog tumor suppressor gene (Yang et al. 2008). Furthermore, studies on human neuroblastoma tissues suggested that miR-184 may rather be proapoptotic (Chen \& Stallings 2007). The same miR molecule may thus act both as an oncogene or a tumor suppressor and may have opposite actions in different tissues. These findings support the tissuespecific nature of miR action and the necessity of tissuespecific target prediction approaches.

As the histological diagnosis of ACC is often difficult, we tried to identify miR markers of malignancy. miR markers have already been shown to be applicable for the differentiation of benign and malignant tumors, e.g. follicular thyroid cancer (Weber et al. 2006). By ROC analysis, $\mathrm{dCT}_{\mathrm{miR}-511}-\mathrm{dCT}_{\mathrm{miR}-503}$ appeared to be the most appropriate marker of malignancy.

We used a novel tissue-specific target prediction approach for deciphering the biological relevance of the six significant miRs in adrenocortical tumors. This tissue-specific target prediction approach is based on the identification of all possible target mRNAs retrieved from three different target prediction algorithms and on the reduction of this huge target mRNA pool in two subsequent steps: i) subtraction of undetectable mRNAs based on microarray lists; ii) search for mRNA targets with inverse alterations in expression as their regulatory $\mathrm{miR}$ molecules. The database-processing software application used in our study was designed to retrieve all possible target mRNAs. The search for inverse alterations in expression was performed by the GSEA software.

Similar approaches for miR target identification have been published recently. Gennarino et al. introduced the software host gene oppositely correlated targets (HOCTAR) that analyzes mRNA profiling data, and looks for inverse expression correlations of genes hosting intragenic miR genes and potential miR target genes. In contrast with our approach, miR expression patterns are not directly analyzed by HOCTAR, and only the expression of miRs whose genes are localized within other genes can be studied (Gennarino et al. 2009).

Huang et al. developed the Bayesian data analysis algorithm, GenMiR ++ , which scores miR-mRNA pairs for inverse expression patterns. mRNA targets were predicted by TargetScan (Huang et al. 2007). Our approach also combines in silico target prediction algorithms with mRNA profiling; however, we used three target prediction algorithms in parallel and subtracted absent mRNAs from the pool of potential mRNA targets. Moreover, Huang's algorithm searches for overexpressed miR and underexpressed mRNA pairs, whereas our approach also investigates pairs of underexpressed miR with overexpressed mRNAs.

Pathway analysis of both tissue-specific miR target mRNA sets and the significant mRNA microarray data revealed damage at $\mathrm{G} 2 / \mathrm{M}$ checkpoint as a major pathogenic pathway in ACC. Cell cycle alterations including damage at $\mathrm{G} 2 / \mathrm{M}$ have been described in the pathogenesis of several tumors (Molinari 2000), and there are some reports describing cell cycle activation in adrenocortical tumors too. The role of G1/S phase acceleration, overexpression of cyclin D1 (CCND1), loss of function of $C D K N 1 C$ ( $p 57^{k i p 2}$ ), and consequent overexpression of cyclin E $(C C N E)$ and G1 cyclindependent kinases (CDK2 and $C D K 4)$ leading to cell cycle progress were found in sporadic ACCs (Bourcigaux et al. 2000, Lombardi et al. 2006). Cyclin B (CCNB1 and $C C N B 2$ ), $C D C 2, C D C 25 B, C D C 25 C$, and $T O P 2 A$ involved in $\mathrm{G} 2 / \mathrm{M}$ transition phase as cell cycle activators were overexpressed in our ACC samples. $C C N B 2$ and TOP $2 A$ overexpression has already been described in ACCs (Giordano et al. 2009). CCNB/ $C D C 2$ complex was found to be overexpressed in 
several malignancies (Egloff et al. 2006); however, its exact role in ACC is unclear. Treatment of ACC cell lines either with $17 \beta$-estradiol $\left(\mathrm{E}_{2}\right)$ and progesterone (Brown et al. 2008) or with the adrenolytic compound mitotane combined with irradiation therapy (Cerquetti et al. 2008) induced G2/M block and apoptosis. Progesterone alone and in combination with $\mathrm{E}_{2}$ induced mitotic perturbations in $\mathrm{SW}-13$ cells via decreased $C C N B 1$ and $C C N D 1$ expression (Brown et al. 2008). By activating cyclin-dependent kinase molecules, $C D C 25 B$ is involved in the pathogenesis of several malignancies (e.g. hepatocellular carcinoma; Yan et al. 2008); however, there are no reports on its role in the pathogenesis of ACC.

In addition, underexpression of Reprimo (RPRM), which is regulated in a p53-dependent manner and is involved in cell cycle arrest at G2 (Taylor \& Stark 2001), further supports that altered G2/M transition may be involved in the pathogenesis of ACC.

A few of these transcripts have been validated to be targets of miRs in non-adrenal cell lines (MertensTalcott et al. 2007, Bueno et al. 2008, Selbach et al. 2008). Based on our results, G2/M checkpoint regulation can thus be modified by miRs, and potential regulatory miRs in the adrenal cortex appear to be different from those validated in other tissues and cell cultures. However, it should be emphasized that miR target interactions reported in this study are only bioinformatically predicted interactions that should be further validated.

In conclusion, to our knowledge, this is the first report on miR expression signatures of sporadic adrenocortical neoplasms. We identified novel miR biomarkers suitable for distinguishing ACC from benign tumors with high sensitivity and specificity. As the histological diagnosis of adrenocortical tumors is often uncertain, these biomarkers can be of great help in cases where the assessment of malignancy is difficult. With our complex pathway analysis approach, different canonical pathways characterized by altered gene expression and potentially subjected to $\mathrm{miR}$ regulation were identified. The previously reported experimental data on the expression of cell cycle regulators and our finding that G2/M checkpoint damage has been revealed by both IPA approaches seem to support the feasibility of our tissue-specific target prediction approach.

\section{Declaration of interest}

The authors declare that there is no conflict of interest that could be perceived as prejudicing the impartiality of the research reported.

\section{Funding}

This work was supported by the Hungarian Scientific Research Fund (OTKA, PD72306); Semmelweis University, the Hungarian Ministry of Health (ETT, 089/2006); Swiss National Fund (FNRS, 3200BO-105657/1); and Fondation pour la Recherche en Endocrinologie, Diabétologie et Métabolisme. A Patócs is a recipient of a Bolyai postdoctoral research fellowship.

\section{Authors contribution statement}

P Igaz designed research; Z Tömböl, P M Szabó, V Molnár, Z Wiener, G Tölgyesi, and I Likó performed research; J Horányi and $\mathrm{P}$ Riesz supplied tissue samples; Z Tömböl, P M Szabó, V Molnár, P Reismann, A Patócs, R-C Gaillard, A Falus, K Rácz, and P Igaz analyzed data; and Z Tömböl, A Patócs, and $\mathrm{P}$ Igaz wrote the paper.

\section{Acknowledgements}

The authors would like to thank Tamás Tömböl dipl. eng. for his help in the development of the data-processing software.

\section{References}

Ambros V, Bartel B, Bartel DP, Burge CB, Carrington JC, Chen X, Dreyfuss G, Eddy SR, Griffiths-Jones S, Marshall M et al. 2003 A uniform system for microRNA annotation. RNA 9 277-279.

Baek D, Villén J, Shin C, Camargo FD, Gygi SP \& Bartel DP 2008 The impact of microRNAs on protein output. Nature 455 64-71.

Barbarotto E, Schmittgen TD \& Calin GA 2008 MicroRNAs and cancer: profile, profile, profile. International Journal of Cancer 122 969-977.

Bernini GP, Moretti A, Bonadio AG, Menicagli M, Viacava P, Naccarato AG, Iacconi P, Miccoli P \& Salvetti A 2002 Angiogenesis in human normal and pathologic adrenal cortex. Journal of Clinical Endocrinology and Metabolism 87 4961-4965.

Bottoni A, Zatelli MC, Ferracin M, Tagliati F, Piccin D, Vignali C, Calin GA, Negrini M, Croce CM \& Degli Uberti EC 2007 Identification of differentially expressed microRNAs by microarray: a possible role for microRNA genes in pituitary adenomas. Journal of Cellular Physiology 210 370-377.

Bourcigaux N, Gaston V, Logié A, Bertagna X, Le Bouc Y \& Gicquel C 2000 High expression of cyclin E and G1 CDK and loss of function of $\mathrm{p} 57^{\mathrm{KIP} 2}$ are involved in proliferation of malignant sporadic adrenocortical tumors. Journal of Clinical Endocrinology and Metabolism 85 322-330.

Brown JW, Prieto LM, Perez-Stable C, Montoya M, Cappell S \& Fishman LM 2008 Estrogen and progesterone lower cyclin B1 and D1 expression, block 
cell cycle in G2/M, and trigger apoptosis in human adrenal carcinoma cell cultures. Hormone and Metabolic Research 40 306-310.

Bueno MJ, de Castro IP \& Malumbres M 2008 Control of cell proliferation pathways by microRNAs. Cell Cycle 7 3143-3148.

Camps C, Buffa FM, Colella S, Moore J, Sotiriou C, Sheldon H, Harris AL, Gleadle JM \& Ragoussis J 2008 Hsa-miR-210 is induced by hypoxia and is an independent prognostic factor in breast cancer. Clinical Cancer Research 14 1340-1348.

Cerquetti L, Bucci B, Marchese R, Misiti S, De Paula U, Miceli R, Muleti A, Amendola D, Piergrossi P, Brunetti E et al. 2008 Mitotane increases the radiotherapy inhibitory effect and induces G2-arrest in combined treatment on both H295R and SW13 adrenocortical cell lines. Endocrine-Related Cancer 15 623-634.

Chen CZ 2005 MicroRNAs as oncogenes and tumor suppressors. New England Journal of Medicine $\mathbf{3 5 3}$ 1768-1771.

Chen Y \& Stallings RL 2007 Differential patterns of microRNA expression in neuroblastoma are correlated with prognosis, differentiation, and apoptosis. Cancer Research 67 976-983.

Cheng AM, Byrom MW, Shelton J \& Ford LP 2005 Antisense inhibition of human miRNAs and indications for an involvement of miRNA in cell growth and apoptosis. Nucleic Acids Research 33 1290-1297.

Egloff AM, Vella LA \& Finn OJ 2006 Cyclin B1 and other cyclins as tumor antigens in immunosurveillance and immunotherapy of cancer. Cancer Research 66 6-9.

Gennarino VA, Sardiello M, Avellino R, Meola N, Maselli V, Anand S, Cutillo L, Ballabio A \& Banfi S 2009 MicroRNA target prediction by expression analysis of host genes. Genome Research 19 481-490.

Giordano TJ, Kuick R, Else T, Gauger PG, Vinco M, Bauersfeld J, Sanders D, Thomas DG, Doherty G \& Hammer G 2009 Molecular classification and prognostication of adrenocortical tumors by transcriptome profiling. Clinical Cancer Research 15 668-676.

Griffiths-Jones S, Saini HK, van Dongen S \& Enright AJ 2008 miRBase: tools for microRNA genomics. Nucleic Acids Research 36 D154-D158.

Grimson A, Farh KK, Johnston WK, Garrett-Engele P, Lim LP \& Bartel DP 2007 MicroRNA targeting specificity in mammals: determinants beyond seed pairing. Molecular Cell 27 91-105.

Grosshans H \& Filipowicz W 2008 Proteomics joins the search for microRNA targets. Cell 134 560-562.

Huang JC, Babak T, Corson TW, Chua G, Khan S, Gallie BL, Hughes TR, Blencowe BJ, Frey BJ \& Morris QD 2007 Using expression profiling data to identify human microRNA targets. Nature Methods 4 1045-1049.

Hulshizer R \& Blalock EM 2007 Post hoc pattern matching: assigning significance to statistically defined expression patterns in single channel microarray data. $B M C$ Bioinformatics 8240.
Igaz P, Wiener Z, Szabo P, Falus A, Gaillard RC, Horanyi J, Racz K \& Tulassay Z 2006 Functional genomics approaches for the study of sporadic adrenal tumor pathogenesis: clinical implications. Journal of Steroid Biochemistry and Molecular Biology 101 87-96.

Koch CA, Pacak K \& Chrousos GP 2002 The molecular pathogenesis of hereditary and sporadic adrenocortical and adrenomedullary tumors. Journal of Clinical Endocrinology and Metabolism 87 5367-5384.

Krek A, Grün D, Poy MN, Wolf R, Rosenberg L, Epstein EJ, MacMenamin P, da Piedade I, Gunsalus KC, Stoffel M et al. 2005 Combinatorial microRNA target predictions. Nature Genetics 37 495-500.

Ladeiro Y, Couchy G, Balabaud C, Bioulac-Sage P, Pelletier L, Rebouissou S \& Zucman-Rossi J 2008 MicroRNA profiling in hepatocellular tumors is associated with clinical features and oncogene/tumor suppressor gene mutations. Hepatology 47 1955-1963.

Libè R, Fratticci A \& Bertherat J 2007 Adrenocortical cancer: pathophysiology and clinical management. Endocrine-Related Cancer 14 13-28.

Lim LP, Lau NC, Garrett-Engele P, Grimson A, Schelter JM, Castle J, Bartel DP, Linsley PS \& Johnson JM 2005 Microarray analysis shows that some microRNAs downregulate large numbers of target mRNAs. Nature 433 769-773.

Livak KJ \& Schmittgen TD 2001 Analysis of relative gene expression data using real-time quantitative PCR and the $2^{-\Delta \Delta \mathrm{C}_{\mathrm{t}}}$ method. Methods 25 402-408.

Lombardi CP, Raffaelli M, Pani G, Maffione A, Princi P, Traini E, Galeotti T, Rossi ED, Fadda G \& Bellantone R 2006 Gene expression profiling of adrenal cortical tumors by cDNA macroarray analysis. Results of a preliminary study. Biomedicine \& Pharmacotherapy 60 186-190.

Mertens-Talcott SU, Chintharlapalli S, Li X \& Safe S 2007 The oncogenic microRNA-27a targets genes that regulate specificity protein transcription factors and the G2/M checkpoint in MDA-MB-231 breast cancer cells. Cancer Research 67 11001-11011.

Molinari M 2000 Cell cycle checkpoints and their inactivation in human cancer. Cell Proliferation $\mathbf{3 3}$ 261-274.

Pavlidis P 2003 Using ANOVA for gene selection from microarray studies of the nervous system. Methods $\mathbf{3 1}$ 82-89.

Resnick KE, Alder H, Hagan JP, Richardson DL, Croce CM \& Cohn DE 2009 The detection of differentially expressed microRNAs from the serum of ovarian cancer patients using a novel real-time PCR platform. Gynecologic Oncology 112 55-59.

Roldo C, Missiaglia E, Hagan JP, Falconi M, Capelli P, Bersani S, Calin GA, Volinia S, Liu CG, Scarpa A et al. 2006 MicroRNA expression abnormalities in pancreatic endocrine and acinar tumors are associated with distinctive pathologic features and clinical behavior. Journal of Clinical Oncology 24 4677-4684. 
Selbach M, Schwanhäusser B, Thierfelder N, Fang Z, Khanin R \& Rajewsky N 2008 Widespread changes in protein synthesis induced by microRNAs. Nature 455 58-63.

Soon PS, McDonald KL, Robinson BG \& Sidhu SB 2008 Molecular markers and the pathogenesis of adrenocortical cancer. Oncologist 13 548-561.

Subramanian A, Tamayo P, Mootha VK, Mukherjee S, Ebert BL, Gillette MA, Paulovich A, Pomeroy SL, Golub TR, Lander ES et al. 2005 Gene set enrichment analysis: a knowledge-based approach for interpreting genome-wide expression profiles. PNAS $\mathbf{1 0 2}$ 15545-15550.

Taylor WR \& Stark GR 2001 Regulation of the G2/M transition by p53. Oncogene 20 1803-1815.

Weber F, Teresi RE, Broelsch CE, Frilling A \& Eng C 2006 A limited set of human microRNA is deregulated in follicular thyroid carcinoma. Journal of Clinical Endocrinology and Metabolism 91 3584-3591.
Wijnhoven BP, Michael MZ \& Watson DI 2007 MicroRNAs and cancer. British Journal of Surgery 94 23-30.

Wong TS, Liu XB, Wong BY, Ng RW, Yuen AP \& Wei WI 2008 Mature miR-184 as potential oncogenic microRNA of squamous cell carcinoma of tongue. Clinical Cancer Research 14 2588-2592.

Yan X, Chua MS, He J \& So SK 2008 Small interfering RNA targeting CDC25B inhibits liver tumor growth in vitro and in vivo. Molecular Cancer 719.

Yang H, Kong W, He L, Zhao JJ, O’Donnell JD, Wang J, Wenham RM, Coppola D, Kruk PA, Nicosia SV et al. 2008 MicroRNA expression profiling in human ovarian cancer: miR-214 induces cell survival and cisplatin resistance by targeting PTEN. Cancer Research $\mathbf{6 8}$ 425-433.

Zhao JJ, Yang J, Lin J, Yao N, Zhu Y, Zheng J, Xu J, Cheng JQ, Lin JY \& Ma X 2009 Identification of miRNAs associated with tumorigenesis of retinoblastoma by miRNA microarray analysis. Child's Nervous System 25 13-20. 